\title{
PSA Testing Use and Prostate Cancer Diagnostic Stage After the 2012 U.S. Preventive Services Task Force Guideline Changes
}

Christopher J. Magnani, MPhila; Kevin Li, BSa; Tina Seto, MS; Kathryn M. McDonald, PhDc; Douglas W. Blayney, MDc,d; James D. Brooks, MDe; and Tina Hernandez-Boussard, PhD, MPH, MSc,f

\begin{abstract}
Background: Most patients with prostate cancer are diagnosed with low-grade, localized disease and may not require definitive treatment. In 2012, the U.S. Preventive Services Task Force (USPSTF) recommended against prostate cancer screening to address overdetection and overtreatment. This study sought to determine the effect of guideline changes on prostate-specific antigen (PSA) screening and initial diagnostic stage for prostate cancer. Patients and Methods: A difference-in-differences analysis was conducted to compare changes in PSA screening (exposure) relative to cholesterol testing (control) after the 2012 USPSTF guideline changes, and chisquare test was used to determine whether there was a subsequent decrease in early-stage, low-risk prostate cancer diagnoses. Data were derived from a tertiary academic medical center's electronic health records, a national commercial insurance database (OptumLabs), and the SEER database for men aged $\geq 35$ years before (2008-2011) and after (2013-2016) the guideline changes. Results: In both the academic center and insurance databases, PSA testing significantly decreased for all men compared with the control. The greatest decrease was among men aged 55 to 74 years at the academic center and among those aged $\geq 75$ years in the commercial database. The proportion of early-stage prostate cancer diagnoses $(<\mathrm{T} 2)$ decreased across age groups at the academic center and in the SEER database. Conclusions: In primary care, PSA testing decreased significantly and fewer prostate cancers were diagnosed at an early stage, suggesting provider adherence to the 2012 USPSTF guideline changes. Longterm follow-up is needed to understand the effect of decreased screening on prostate cancer survival.
\end{abstract}

J Natl Compr Canc Netw 2019;17(7):795-803 doi: 10.6004/jnccn.2018.7274

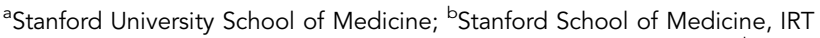
Research Technology; 'Department of Medicine, Stanford University; ${ }^{\text {'SStanford }}$ Cancer Institute; and ${ }^{\circ}$ Department of Urology and ${ }^{\mathrm{D} D e p a r t m e n t}$ of Biomedical Data Science, Stanford University, Stanford, California.

\section{Background}

Prostate cancer is the most common cancer in US men; in 2019, 174,700 new cases are estimated, accounting for $20 \%$ of all new cancers in men, with 31,700 deaths. ${ }^{1}$ However, a lack of consensus remains regarding best practices for screening and treatment, partly because of the difficulty in distinguishing aggressive from indolent cancers. $^{2}$ Most prostate cancers are asymptomatic, are detected by primary care-directed screening, are slowgrowing, and will not become clinically evident during the patient's lifetime. Autopsy studies detect prostate cancer in $30 \%$ of men by age 55 years and $60 \%$ of men by age 80 years. ${ }^{3}$ Widespread implementation of prostatespecific antigen (PSA) screening has led to a significant increase in diagnosis and treatment of prostate cancer, including many inconsequential tumors, ${ }^{4}$ with minimal or no effect on mortality rates. ${ }^{5-9}$ Meanwhile, treatment of these cancers can lead to treatment-related adverse events, such as urinary incontinence or sexual dysfunction..$^{10,11}$

The Prostate, Lung, Colorectal and Ovarian Cancer Screening Trial (PLCO) showed that systematic PSA testing resulted in higher prostate cancer diagnosis rates, particularly of early-stage disease, but without improvements in mortality. ${ }^{7}$ In addition, the Prostate Cancer Intervention Versus Observation Trial (PIVOT) showed no survival advantage for surgery compared with no treatment in patients with localized prostate cancer. ${ }^{12}$ Based on the results of these trials, the U.S. Preventive Services Task Force (USPSTF) published new guidelines in 2012 recommending against PSA screening in all men (D rating), ${ }^{13}$ expanding on a 2008 recommendation against screening in men aged $\geq 75$ years. ${ }^{14}$ However, these recommendations were highly controversial because the death rate of prostate cancer had decreased $50 \%$ since the initiation of PSA testing in the United States, and

See JNCCN.org for supplemental online content. 
randomized trials of PSA screening and surgical treatment of localized prostate cancer conducted in Europe showed significant survival benefits for both screening and treatment. ${ }^{5,15,16}$ The controversy continued to increase based on criticisms of PLCO citing contamination of the control $\mathrm{arm}^{17}$ and criticisms of PIVOT for selection of patients with comorbidities and indolent disease. ${ }^{18}$ Finally, since 2012, the increased use of active surveillance for management of indolent disease in both the United States and Europe changed the risk/benefit ratio for prostate cancer screening by decoupling screening from treatment-related adverse events. ${ }^{19}$ Because of this, in 2018, the USPSTF rolled back the 2012 recommendations and advised men aged 55 to 69 years to discuss the risks and benefits of screening with their healthcare providers $\left(\mathrm{C}\right.$ rating). ${ }^{20}$

A better understanding of the effects of guideline changes, particularly regarding controversial topics such as cancer screening, may help inform future policy. Studies of Medicare beneficiaries have shown that the 2008 guideline changes were associated with a $2 \%$ decline in PSA screening for men aged $\geq 75$ years and a decline in treatment by $42 \%$ at the population level but only by $8 \%$ among diagnosed men, suggesting that declines in screening and diagnosis were driving the decline rather than changes in treatment patterns. ${ }^{21}$ Studies examining men of all ages have found conflicting results, observing significant declines ${ }^{22,23}$ or no change ${ }^{24}$ in PSA screening rates in the wake of the 2012 changes, and declines in testing have been suggested to underlie increases in late-stage disease burden from 2010 to $2014 .^{23}$ However, these studies did not control for secular trends, such as the Affordable Care Act (ACA), that might influence screening and diagnosis, and encompassed only 1 or 2 years of data after the guideline changes.

Given the controversy about this guideline change, clinician adherence and effects on prostate cancer diagnosis are poorly understood. Using multiple datasets from 2008 to 2016, this study sought to determine whether PSA testing rates changed in primary care after the 2012 USPSTF guideline changes and whether earlystage, low-risk prostate cancer diagnoses decreased after the downgrade in PSA screening recommendations. Our findings highlight the impact even controversial guideline changes can have on clinical practice.

\section{Patients and Methods}

\section{Study Design}

A quasiexperimental, difference-in-differences (DID) design $^{25}$ was used to compare PSA versus cholesterol testing rates among men aged $\geq 35$ years before (2008-2011; "prepolicy") and after (2013-2016; "postpolicy") the 2012 changes to the USPSTF prostate cancer screening guidelines. We focused on primary care providers because they are tasked with disease screening, whereas subspecialists likely use PSA testing to monitor disease after treatment. Cholesterol testing, like PSA testing, addresses conditions that are asymptomatic at onset, targets similar risk populations, is administered as a blood test, is widely accessible across care settings, and is mainly used by primary care physicians. The DID design allows the control to serve as the counterfactual, thereby accounting for secular trends such as increased access to care after the ACA. We adjusted for potential timevarying confounders that could bias estimates and tested for parallelism in prepolicy trends between the study and control populations before the guideline changes, adhering to published best practices to assess validity of the control as a suitable counterfactual. ${ }^{25,26} \mathrm{We}$ further compared rates of prostate and colorectal cancers (CRCs) diagnosed at an early stage both prepolicy and postpolicy. CRC has a patient population and clinically silent period similar to prostate cancer, yet screening guidelines were stable during the study period.

\section{Data Sources}

Primary data were derived from the electronic health records (EHRs) of a tertiary academic medical center containing encounter-level data from 2008 to 2016, including demographics, laboratory orders, insurance payer, clinical features, and provider specialty. The clinical data warehouse is described elsewhere. ${ }^{27}$

OptumLabs, co-founded by Mayo Clinic and Optum in late 2012, is a commercial data, infrastructure services, and care organization that is part of UnitedHealth Group. OptumLabs now has 30 partners and a HIPAA-compliant deidentified database of $>200$ million people. Records include inpatient, outpatient, pharmacy, and laboratory claims. Socioeconomic status (SES) was established using net worth as coded by the OptumLabs database. We used a $1 \%$ sample of the population from 2008 to $2016 .^{28}$

The SEER Program is a national cancer database encompassing approximately one-third of the US population. We used 2008 to 2015 data for both prostate cancer and CRC diagnoses, including demographics and diagnostic stage. SEER data were available up to 2015 and lacked comorbidity scores. ${ }^{29}$ Insurance was categorized as insured (Medicare or private), any Medicaid, or other/unknown/uninsured.

\section{Study Participants}

The screening population consisted of undiagnosed men aged $\geq 35$ years seen by a primary care provider. Primary care was defined in EHR data by provider specialty (family medicine, family practice, geriatric medicine, or nurse practitioner-family), whereas the OptumLabs database already included a variable for provider type 
that identified records from primary care providers. Charlson comorbidity scores were assigned at the start of each year, and ages were calculated between birth and encounter dates. Race was classified as white, Asian, black, Hispanic, and other/unknown. Insurance payer was categorized as Medicare, Medicaid, private, and other/unknown/ uninsured. Annual testing rates were assessed independently: patients could be counted as receiving screening or not only once per annual eligibility period. Diagnosed patients were excluded after their diagnosis date.

Diagnostic stage was assessed in all first-time cancer diagnoses by calendar year. "Low-grade" was defined based on AJCC prognostic stage groups ${ }^{30}$; "early-stage" was defined as localized cancer (summary stage $\leq 2$ ) at initial diagnosis. Cancers with unrecorded initial stage were excluded.

\section{Statistical Analysis}

Linear regression DID models compared changes in PSA screening relative to cholesterol testing after the 2012 USPSTF recommendation. The models account for secular changes, which include factors such as expanded access to care after the ACA, by assuming the control is a counterfactual for the exposure group had the policy not existed. We adhered to published best practices in assessing this assumption by testing for parallelism in the preintervention period (see supplemental eAppendices 1 and 2, available with this article at JNCCN.org). ${ }^{25,26}$ Linear probability models were a function of separate binary indicator variables for exposure status, postpolicy status (2013-2016), and their product yielding their interaction (supplemental eAppendix 1). The DID estimate is represented by the interaction term, which describes the differential change between exposure and control after policy implementation. Charlson comorbidity score, age, race, and insurance or SES (net worth) were included in the models. The prepolicy period was defined as January 1, 2008, through December 31, 2011, and the postpolicy period as January 1, 2013, through December 31, 2016 (2015 for SEER). The implementation year, 2012, was excluded as a "washout" period. ${ }^{13}$ Screening trends compared PSA (exposure) and cholesterol (control) testing. Diagnostic stage was separately examined for prostate cancer and CRC using chi-square test. We stratified analyses by age group. Statistical significance was defined by a 2 -sided $P$ value $<.05$. All analyses were performed with R 3.4.1 (The R Foundation) and RStudio 1.0.153 (RStudio).

\section{Results}

In the academic center's database, we identified 18,559 prepolicy and 78,281 postpolicy patients; 256 (1.4\%) prepolicy and 874 (1.1\%) postpolicy patients were excluded for prostate cancer diagnosis before annual PSA screening was tabulated. Before the 2012 USPSTF recommendation, 3,252 received any PSA tests $(3,456$ tests ordered) and 5,686 received any cholesterol tests (6,410 tests ordered); after the 2012 USPSTF recommendation, 8,306 patients received any PSA tests $(8,914$ total tests ordered) and 24,491 received any cholesterol tests $(28,161$ total tests ordered). Patients in the postpolicy group were slightly older, had more men aged 55 to 74 years, included slightly fewer on Medicare, and had slightly more black and Hispanic patients, but fewer Asian patients compared with the prepolicy group (Table 1).

In the OptumLabs 1\% sample, we identified 93,334 prepolicy and 110,067 postpolicy patients. Patient counts for the control and exposure groups were equivalent for the OptumLabs analysis because patient records after the date of prostate cancer diagnosis were pre-excluded during the initial data extraction. Postpolicy patients (Table 1) were older, had more men aged 55 to 74 years and fewer aged 35 to 54 years, included fewer white patients but more with other/unknown race, and had more with unknown SES (net worth) compared with prepolicy patients. The number of patients in the academic center's population increased during the course of the study, due to an expanded primary care initiative, which is controlled for along with other background temporal trends through the DID model via the cholesterol control.

Unadjusted trends in annual PSA (exposure) and cholesterol (control) testing in the primary care setting, including composite rates and rates stratified by age group, are shown in Figure 1 for the tertiary academic center and OptumLabs. PSA testing declined in both sites, with the greatest decreases in PSA testing observed in men aged 55 to 74 years and $\geq 75$ years, respectively. Modeled estimates accounting for background temporal trends (Table 2) show significant decreases in PSA testing both overall and by age group (all $P<.001$ ). PSA testing declined across all age groups by $8.0 \%$ (95\% CI, $-8.9 \%$ to $-7.1 \%)$ in the academic center and by $3.6 \%(95 \% \mathrm{CI}$, $-4.1 \%$ to $-3.2 \%$ ) in the OptumLabs population. The academic center had the largest changes in men aged 55 to 74 years $(-13.0 \%)$ and smaller declines in men aged 35 to $54(-4.8 \%)$ and $\geq 75$ years $(-8.5 \%)$. OptumLabs had its largest decrease in men aged $\geq 75$ years $(-8.2 \%)$, with smaller declines in men aged 55 to $74(-2.8 \%)$ and 35 to 54 years $(-4.1 \%)$.

In the academic center database, we identified 2,572 prostate and 413 CRC prepolicy diagnoses after excluding $288(10.1 \%)$ and $204(33.1 \%)$ without stage, respectively, and 1,397 prostate and 521 CRC postpolicy diagnoses after excluding 593 (29.8\%) and 176 (25.3\%) without stage, respectively. Postpolicy patients with prostate cancer had similar age, slightly higher comorbidity scores, more white and Asian patients, and more 
Table 1. Characteristics of Patients Eligible for Screening

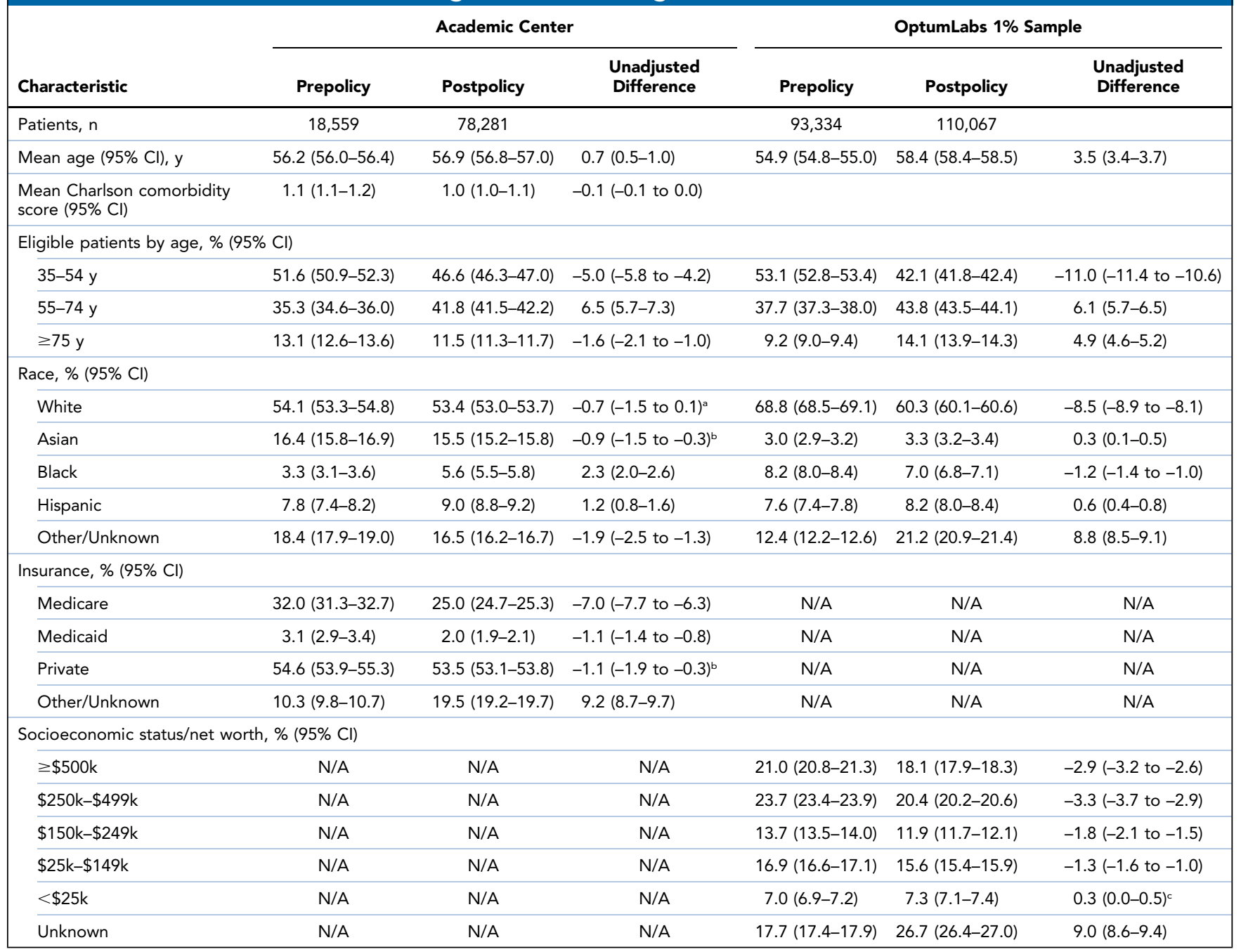

All comparisons are between the prepolicy (2008-2011) and postpolicy (2013-2016) periods in primary care. Time intervals were defined as calendar years and evaluated independently for patient-level eligibility. All $P$ values are $<.001$ except where indicated.

Abbreviation: N/A, not available.

a $P=.094$.

b $P<.01$.

${ }^{c} P=.063$.

patients with Medicaid and private insurance compared with prepolicy patients (supplemental eAppendix 3). Compared to prepolicy patients, postpolicy patients with CRC included fewer white and more Asian men, but there was no statistically significant difference in age, comorbidity, or insurance. In both the prepolicy and postpolicy periods, the prostate cancer group was generally older compared with the CRC group and had fewer Asian patients.

In the SEER sample, we identified 75,641 prostate and 20,250 CRC prepolicy diagnoses after excluding 1,945 (2.5\%) and 821 (3.9\%) without stage, respectively, and 44,904 prostate and 15,077 CRC postpolicy diagnoses after excluding 1,477 (3.2\%) and 681 (4.3\%) without stage, respectively. After the 2012 USPSTF recommendation (postpolicy), age slightly increased for patients with prostate cancer and decreased for those with CRC (supplemental eAppendix 3); both had fewer white individuals and a larger Medicaid proportion postpolicy. Patients with prostate cancer were slightly older than those with CRC in both the prepolicy and postpolicy periods.

Decreases in the unadjusted proportion of earlystage diagnoses (Table 3) were seen in both the academic center and SEER databases. The academic center had nearly uniform decreases across age groups, with an overall decline from $79.0 \%$ to $63.4 \%(-15.6 \%)$. In comparison, CRC diagnoses did not display significant changes, except for an increase from $46.3 \%$ to $58.6 \%$ 
A

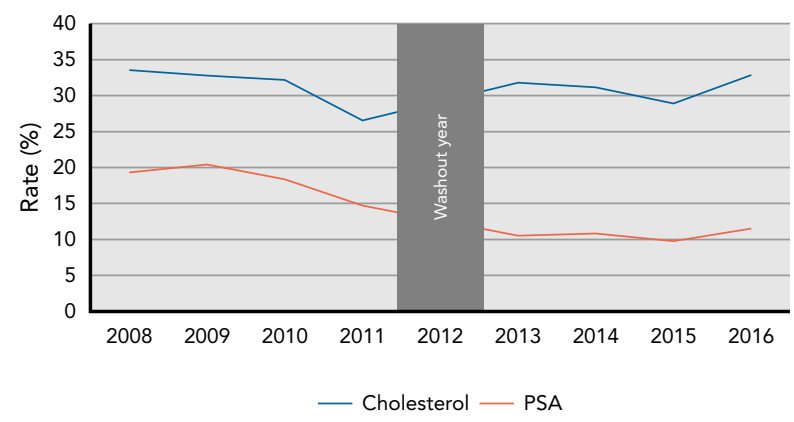

C

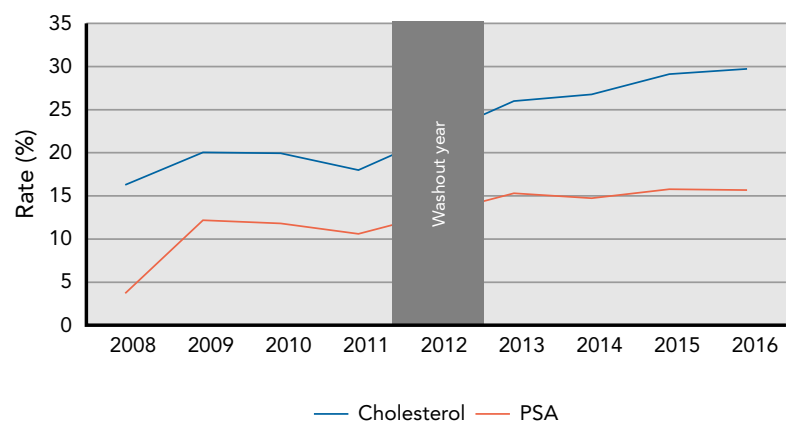

B

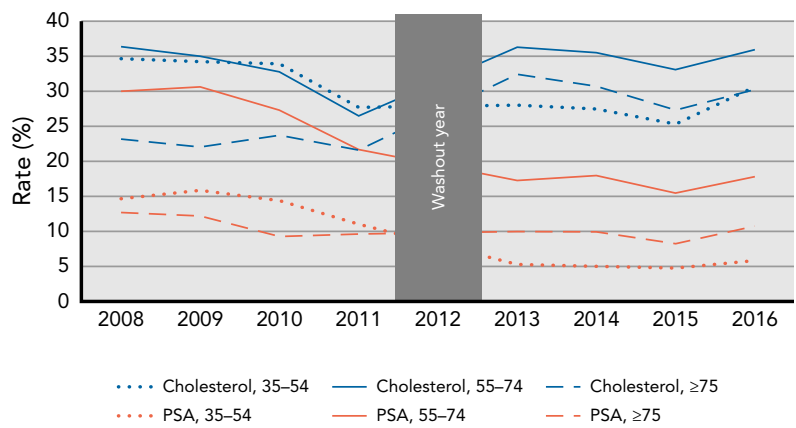

D

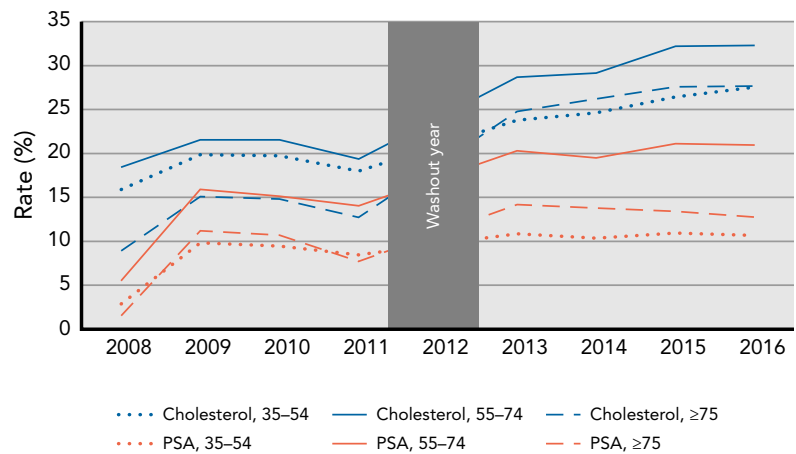

Figure 1. Unadjusted trends in PSA (exposure) and cholesterol (control) testing in (A) academic center, all ages, (B) academic center, by age group, (C) OptumLabs, all ages, and (D) OptumLabs, by age group.

Abbreviation: PSA, prostate-specific antigen.

$(+12.3 \% ; P<.01)$ in men aged 55 to 74 . SEER showed smaller decreases in early-stage prostate cancer, decreasing overall from $82.6 \%$ to $77.7 \%$ ( $-4.9 \%)$, with the largest decline in men aged $\geq 75$ years $(-10.1 \%)$, followed by 55 to 74 years $(-4.1 \%)$ and 35 to 54 years $(-2.6 \%)$. CRC diagnoses also decreased, but to a lesser degree, from $44.0 \%$ to $41.7 \%$ overall $(-2.3 \%$ ), with men aged $\geq 75$ years showing the largest decrease $(-4.0 \%)$, followed by those aged 55 to 74 years $(-2.3 \% ; P<.01)$, whereas men aged 35 to 54 years had no significant change. All results were significant at $P<.001$ unless noted otherwise.

\section{Discussion}

This large, retrospective, observational study found that PSA testing rates in the primary care setting decreased relative to cholesterol screening across age groups in both an academic medical center and a large commercial claims database after the controversial 2012 USPSTF recommendation against PSA screening in men of all ages. The academic center saw the largest decreases among men aged 55 to 74 years-the population that many clinicians view as the target prostate cancer screening population. Although the USPSTF has never endorsed PSA screening, their updated 2018 guidelines upgraded its recommendation from a grade $\mathrm{D}$ to a $\mathrm{C}$ rating for men aged 55 to 69 years, softening an explicit recommendation against screening to one that assigns the decision to patients and their doctors after discussing the risks and benefits. ${ }^{20}$ In the commercial database, the greatest decrease in PSA testing was seen in men aged $\geq 75$ years, reflecting continued improvement in adherence to 2008 USPSTF guidelines. Coinciding with these declines in PSA testing was a decrease in the proportion of patients diagnosed with early-stage prostate cancer, as would be expected based on results of randomized PSA screening trials showing fewer diagnoses in the nonscreened control arm..$^{6,70}$ It is notable that the decline in diagnoses was confined to early-stage cancers, potentially decreasing the number of cancers identified when curable, but also reducing rates of overdiagnosis (and subsequent overtreatment). ${ }^{16}$

These findings show that the guideline changes had durable effects on practice patterns through 2016, in line with previous work showing declines of $3 \%$ to $10 \%$ in PSA screening across age groups with data through $2013 .{ }^{31} \mathrm{~A}$ recent survey study showed that men aged 55 to 59 years, 60 to 74 years, and $\geq 75$ years had similar decreases in screening after the 2012 guidelines, ${ }^{32}$ whereas another 


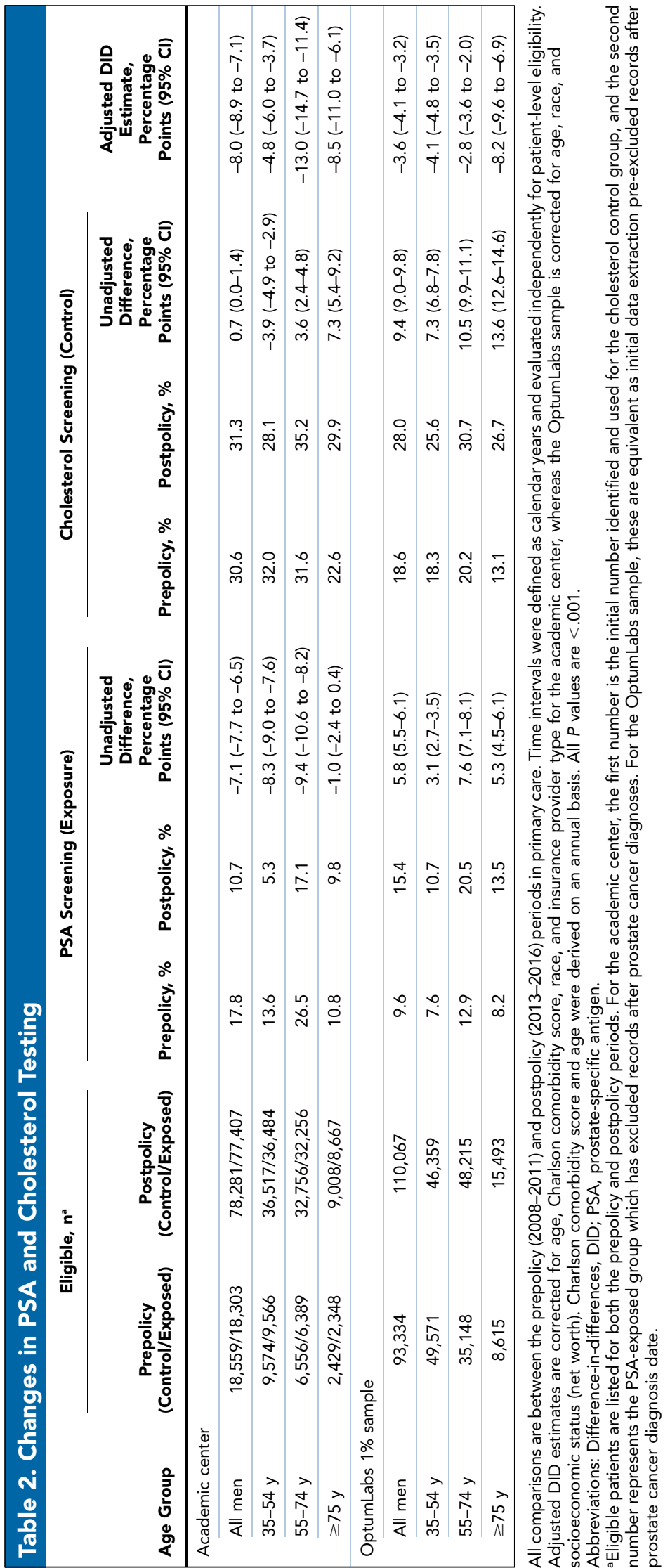




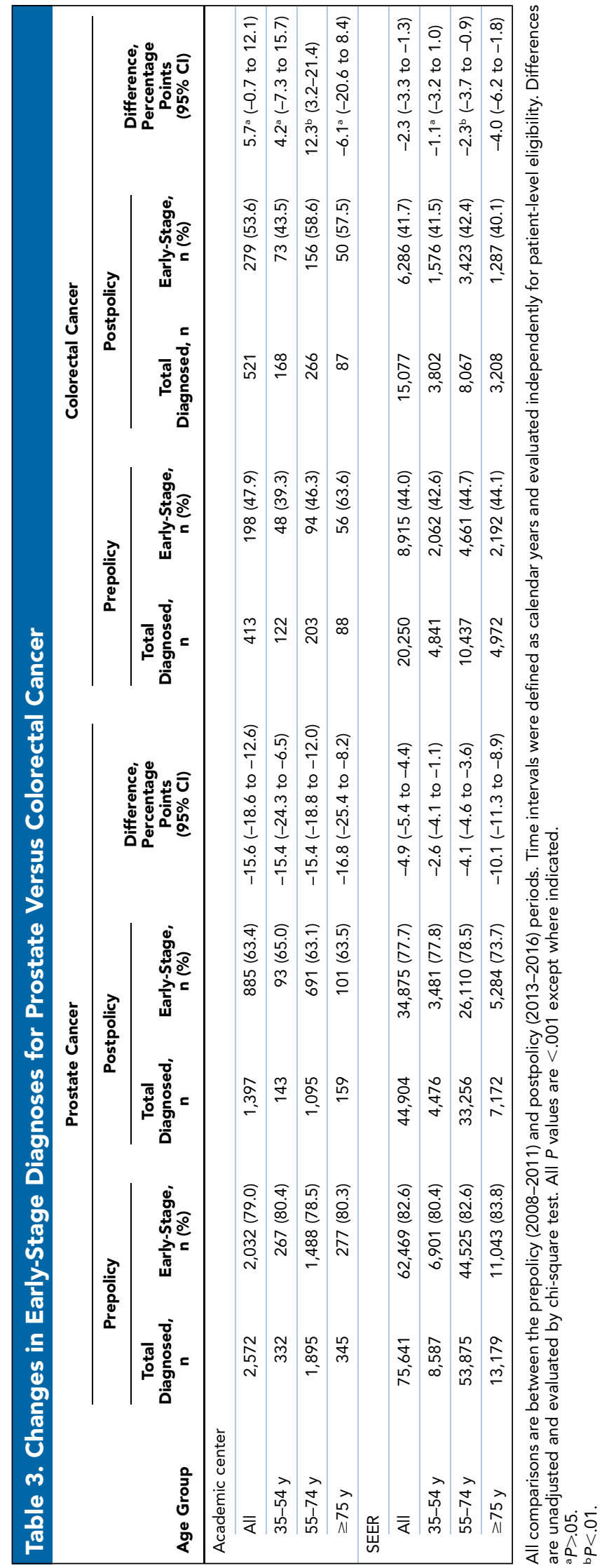

found significant declines of $5 \%$ to $10 \%$ in men aged $<75$ years. ${ }^{33}$ These studies only included data through 2013 - 1 year after the recommendation changes; therefore, our findings show that the guideline changes had durable effects on practice patterns through 2016. An analysis of OptumLab's privately insured patients showed a $38.4 \%$ decline in PSA testing that was restricted to men aged $\geq 75$ years, whereas no significant changes were seen in younger men. Again, this study was limited to data through 2013 and calculated raw rates of PSA testing that did not account for secular trends. ${ }^{34}$ By including data through 2016 and accounting for secular trends through the DID method, we found declines in PSA testing across ages, demonstrating that the declines were generalized and not restricted to an academic practice. It is of interest that the academic center showed greater declines in PSA testing, suggesting a closer adherence to the guidelines. Whether this is true generally or is caused by other factors, such as regional difference in practice patterns, should be tested by analyzing EHR-extracted data in academic and nonacademic settings.

We observed reductions in unadjusted proportions of prostate cancers diagnosed at an early stage, particularly compared with CRCs, showing that the number and proportion of low-risk prostate cancer diagnoses decreased after the guideline changes, consistent with ongoing attempts to reduce overtreatment. ${ }^{11,19,35,36}$ Data from SEER have shown that prostate cancer incidence as a whole has declined substantially in recent years, ${ }^{31}$ including early-stage cancers, although the decrease in early-stage cancers has attenuated since 2013. ${ }^{37}$ Our study included more years after the guideline changes, and our results suggest continued but modest declines in the proportion of early-stage cancers. Future work will need to evaluate whether the 2018 upgrade of the recommendations to a $\mathrm{C}$ rating ${ }^{20}$ will affect PSA testing rates.

Our findings suggest that guideline changes affect physician behavior, at least in terms of the controversial recommendations regarding prostate cancer screening. In addition, the decrease in diagnosis of early-stage prostate cancer shows that the guidelines affect important disease characteristics in patients. Fewer early-stage cancers will decrease the number of men overtreated for prostate cancer and thereby diminish the burden of adverse effects associated with treatment, such as incontinence and erectile dysfunction. ${ }^{10,11,38,39}$ However, some randomized trials of screening and treatment have shown mortality reductions in screened populations and among men treated, as opposed to observed, for early-stage prostate cancer. ${ }^{5,15}$ Because prostate cancer has a long natural history, changes in mortality rates in the population might not be seen for several years after guideline changes. 
Our study has limitations that should be mentioned. First, we used a nonrandomized design and thus could not prove that the 2012 USPSTF recommendations caused any of the observed changes in PSA testing; however, studying changes over time, using multiple datasets, and controlling for patient demographics reduced the chance that the results were confounded by variations in unobserved patient characteristics. Our analysis assumed that cholesterol testing patterns serve as a counterfactual that reflects secular changes in practice patterns, because cholesterol testing is widely practiced, administered via blood test, and applied in men across a spectrum of ages, and guidelines regarding its use did not change during the study period. We followed published best practices in assessing this assumption by testing for parallelism in the prepolicy period. ${ }^{25,26}$ If this assumption was inaccurate, the results of our analysis could be biased.

\section{Conclusions}

After the USPSTF's 2012 grade D recommendation against PSA screening, we observed declines in primary care PSA testing rates relative to cholesterol testing patterns and a decrease in the proportion of prostate cancers diagnosed at an early stage. Our study shows that primary care physicians respond to guideline changes by changing their practice patterns. Guideline adherence was not absolute, evidenced by the continued use of PSA testing, and likely reflects controversies surrounding PSA testing. Our findings show that as the healthcare system moves to a more efficient, patient-centered focus and guidelines and quality metrics become widespread, providers are rapidly responding to guideline recommendations. However, advances in screening technologies are likely to beget clinical scenarios that parallel the controversies surrounding PSA testing. It is important to consider how best to meet needs for disease screening in adults and the efficient use of health services. Further research is needed to understand the effects of the USPSTF's guideline changes on cancer survival.

Submitted September 15, 2018; accepted for publication January 15, 2019.

Author contributions: Study concept and design: All authors. Data acquisition analysis, or interpretation: Magnani, Seto, Hernandez-Boussard. Statistical analysis: Magnani, Hernandez-Boussard. Administrative, technical, or material support: Hernandez-Boussard. Supervision: Hernandez-Boussard. Drafting of manuscript: Magnani. Critical revision: All authors.

Disclosures: The authors have not received any financial consideration from any person or organization to support the preparation, analysis, results, or discussion of this article.

Correspondence: Tina Hernandez-Boussard, PhD, MPH, MS, Stanford University School of Medicine, 1265 Welch Road, Stanford, CA 94305-5479. Email: boussard@stanford.edu

\section{References}

1. Siegel RL, Miller KD, Jemal A. Cancer statistics, 2019. CA Cancer J Clin 2019;69:7-34.

2. Ghani KR, Miller DC. Variation in prostate cancer care. JAMA 2015;313: 2066-2067.

3. Bell KJ, Del Mar C, Wright G, et al. Prevalence of incidental prostate cancer: a systematic review of autopsy studies. Int J Cancer 2015;137: 1749-1757.

4. Roach M III, Thomas K. Overview of randomized controlled treatment trials for clinically localized prostate cancer: implications for active surveillance and the United States Preventive Task Force report on screening? J Natl Cancer Inst Monogr 2012;2012:221-229.

5. Schröder FH, Hugosson J, Roobol MJ, et al. Screening and prostate cancer mortality: results of the European Randomised Study of Screening for Prostate Cancer (ERSPC) at 13 years of follow-up. Lancet 2014;384: 2027-2035.

6. van Leeuwen PJ, Kranse R, Hakulinen T, et al. Impacts of a populationbased prostate cancer screening programme on excess total mortality rates in men with prostate cancer: a randomized controlled trial. J Med Screen 2013;20:33-38.

7. Andriole GL, Crawford ED, Grubb RL, et al. Mortality results from a randomized prostate-cancer screening trial. N Engl J Med 2009;360: 1310-1319.

8. Pinsky PF, Prorok PC, Yu K, et al. Extended mortality results for prostate cancer screening in the PLCO trial with median follow-up of 15 years. Cancer 2017;123:592-599.

9. Martin RM, Donovan JL, Turner EL, et al. Effect of a low-intensity PSAbased screening intervention on prostate cancer mortality: the CAP randomized clinical trial. JAMA 2018;319:883-895.

10. Stanford JL, Feng Z, Hamilton AS, et al. Urinary and sexual function after radical prostatectomy for clinically localized prostate cancer: the Prostate Cancer Outcomes Study. JAMA 2000;283:354-360.

11. Wilt TJ, MacDonald R, Rutks I, et al. Systematic review: comparative effectiveness and harms of treatments for clinically localized prostate cancer. Ann Intern Med 2008;148:435-448.
12. Wilt TJ, Brawer MK, Jones KM, et al. Radical prostatectomy versus observation for localized prostate cancer. N Engl J Med 2012;367:203-213.

13. Moyer VA; U.S. Preventive Services Task Force. Screening for prostate cancer: U.S. Preventive Services Task Force recommendation statement. Ann Intern Med 2012;157:120-134.

14. Lin K, Lipsitz R, MillerT, et al. Benefits and harms of prostate-specific antigen screening for prostate cancer: an evidence update for the U.S. Preventive Services Task Force. Ann Intern Med 2008;149:192-199.

15. Bill-Axelson A, Holmberg L, Garmo H, et al. Radical prostatectomy or watchful waiting in early prostate cancer. N Engl J Med 2014;370:932-942.

16. Brooks JD. Managing localized prostate cancer in the era of prostatespecific antigen screening. Cancer 2013;119:3906-3909.

17. Shoag JE, Mittal S, Hu JC. Reevaluating PSA testing rates in the PLCO trial. N Engl J Med 2016;374:1795-1796.

18. Barbosa PV, Thomas IC, Srinivas $S$, et al. Overall survival in patients with localized prostate cancer in the US Veterans Health Administration: is PIVOT generalizable? Eur Urol 2016;70:227-230.

19. Weiner $A B$, Patel SG, Etzioni R, et al. National trends in the management of low and intermediate risk prostate cancer in the United States. J Urol 2015;193:95-102.

20. Grossman DC, Curry SJ, Owens DK, et al. Screening for prostate cancer: US Preventive Services Task Force recommendation statement. JAMA 2018;319:1901-1913.

21. Borza T, Kaufman SR, Shahinian VB, et al. Sharp decline in prostate cancer treatment among men in the general population, but not among diagnosed men. Health Aff (Millwood) 2017;36: 108-115.

22. Jemal A, Fedewa SA, Ma J, et al. Prostate cancer incidence and PSA testing patterns in relation to USPSTF screening recommendations. JAMA 2015;314:2054-2061.

23. Negoita S, Feuer EJ, Mariotto A, et al. Annual report to the nation on the status of cancer, part II: recent changes in prostate cancer trends and disease characteristics. Cancer 2018;124:2801-2814. 
24. Hutchinson R, Akhtar A, Haridas J, et al. Testing and referral patterns in the years surrounding the US Preventive Services Task Force recommendation against prostate-specific antigen screening. Cancer 2016;122: 3785-3793.

25. Dimick JB, Ryan AM. Methods for evaluating changes in health care policy: the difference-in-differences approach. JAMA 2014;312: 2401-2402

26. Ryan AM, Burgess JF Jr, Dimick JB. Why we should not be indifferent to specification choices for difference-in-differences. Health Serv Res 2015; 50:1211-1235.

27. Seneviratne MG, Seto T, Blayney DW, et al. Architecture and implementation of a clinical research data warehouse for prostate cancer. EGEMS (Wash DC) 2018;6:13.

28. Wallace PJ, Shah ND, Dennen T, et al. Optum Laboratories: building a novel node in the learning health care system. Health Aff (Millwood) 2014; 33:1187-1194.

29. Surveillance, Epidemiology, and End Results (SEER) Program (www.seer.cancer.gov) Research Data (1975-2015), National Cancer Institute, DCCPS, Surveillance Research Program, released April 2018 , based on the November 2017 submission.

30. Amin MB, Edge S, Greene F, et al, eds. AJCC Cancer Staging Manual. 8th ed. Chicago, IL: American College of Surgeons; 2018.

31. Fleshner K, Carlsson SV, Roobol MJ. The effect of the USPSTF PSA screening recommendation on prostate cancer incidence patterns in the USA. Nat Rev Urol 2017;14:26-37.
32. Drazer MW, Huo D, Eggener SE. National prostate cancer screening rates after the 2012 US Preventive Services Task Force recommendation discouraging prostate-specific antigen-based screening. J Clin Oncol 2015 33:2416-2423.

33. Sammon JD, Abdollah F, Choueiri TK, et al. Prostate-specific antigen screening after 2012 US Preventive Services Task Force recommendations. JAMA 2015;314:2077-2079.

34. Kim SP, Karnes RJ, Gross CP, et al. Contemporary national trends of prostate cancer screening among privately insured men in the United States. Urology 2016;97:111-117.

35. Gandaglia G, Briganti A, Fossati N, et al. The problem is not what to do with indolent and harmless prostate cancer-the problem is how to avoid finding these cancers. Eur Urol 2016;70:547-548.

36. Polascik TJ, Passoni NM, Villers A, et al. Modernizing the diagnostic and decision-making pathway for prostate cancer. Clin Cancer Res 2014;20: 6254-6257

37. Jemal A, Ma J, Siegel R, et al. Prostate cancer incidence rates 2 years after the US Preventive Services Task Force recommendations against screening. JAMA Oncol 2016;2:1657-1660.

38. Potosky AL, Legler J, Albertsen PC, et al. Health outcomes after prostatectomy or radiotherapy for prostate cancer: results from the Prostate Cancer Outcomes Study. J Natl Cancer Inst 2000;92:1582-1592.

39. Hamdy FC, Donovan JL, Lane JA, et al. 10-Year outcomes after monitoring, surgery, or radiotherapy for localized prostate cancer. $N$ Engl J Med 2016;375:1415-1424.

\section{NCCN $\begin{aligned} & \text { National Comprehensive } \\ & \text { Cancer Network }{ }^{\circledR}\end{aligned}$}

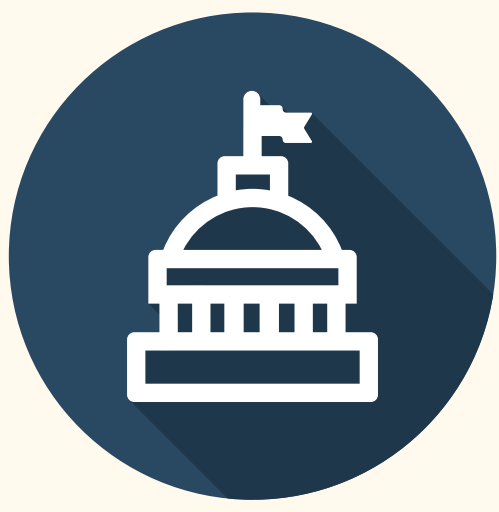

\section{Defining and Applying Quality in an Evolving Health Policy Landscape and the Implications for Cancer Care}

\author{
September 12,2019 \\ The National Press Club \\ Washington, DC
}

\section{Visit NCCN.org/policy to register}

Supported by Adaptive Biotechnologies; AmerisourceBergen; Apobiologix; Astellas; AstraZeneca through its NCCN Corporate Council Membership; Bristol-Myers Squibb; Celgene Corporation; Eisai Inc.; EMD Serono; Ferring Pharmaceuticals; Foundation Medicine; HELSINN; Heron; Incyte Corporation; Kite, a Gilead Company; an independent educational grant from Merck \& Co., Inc.; Novartis; Regeneron Pharmaceuticals Inc; Tesaro; Verastem Oncology. Sponsored by Boehringer Ingelheim Pharmaceuticals Inc; Dendreon Pharmaceuticals LLC; Janssen Oncology, Pharmaceutical Companies of Johnson \& Johnson; This activity was made possible by Pharmacyclics, an AbbVie Company; This activity is supported by a contribution from Lilly. 
Supplemental online content for:

\section{PSA Testing Use and Prostate Cancer Diagnostic Stage After the 2012 U.S. Preventive Services Task Force Guideline Changes}

Christopher J. Magnani, MPhil; Kevin Li, BS; Tina Seto, MS; Kathryn M. McDonald, PhD; Douglas W. Blayney, MD; James D. Brooks, MD; and Tina Hernandez-Boussard, PhD, MPH, MS

J Natl Compr Canc Netw 2019;17(7):795-803

eAppendix 1: Supplemental Methods: Regression Equations

eAppendix 2: Prepolicy Trends in Prostate-Specific Antigen and Cholesterol Testing in the Primary Care Setting eAppendix 3: Characteristics of Diagnosed Patient Population 


\section{eAppendix 1. Supplemental Methods: Regression Equations}

\section{Difference-in-Differences Analysis}

$$
\begin{aligned}
\text { Outcome }_{\text {itk }}= & \beta_{o}+\beta_{1} \text { Postpolicy }_{t}+\beta_{2} \text { Exposed }_{k}+\beta_{3} \text { Postpolicy }_{t} \text { Exposed }_{k}+\gamma_{1} \text { Race }_{i}+\gamma_{2} \text { Age }_{i} \\
& +\gamma_{3} \text { Insurance }_{i}+\gamma_{4} \text { CharlsonComorbidity }_{i}+\varepsilon_{i c t}
\end{aligned}
$$

(1)

Indexed patient, time period (eligibility interval), and cohort (exposed $=$ prostate) are represented by $i, t$, and $k$, respectively. Postpolicy was an indicator for the time period after implementation of the U.S. Preventive Services Task Force guideline changes (2013-2016). Exposed was an indicator for the cohort eligible for prostate-specific antigen (exposure) or cholesterol (control) testing. Potential confounders were race (white, Asian, black, Hispanic, other), age, insurance (Medicare, Medicaid, private, other), and Charlson comorbidity score. $\beta_{3}$ is the difference-in-differences estimate, which provides the differential change between the exposure and control groups after policy implementation.

\section{Parallel Trends Assumption: Testing for Prepolicy (2008-2011) Trend Divergence of Exposure and Control}

$$
\begin{aligned}
\text { Outcome }_{i t k}= & \beta_{o}+\beta_{1} \text { TimeTrend }_{t}+\beta_{2} \text { Exposed }_{k}+\beta_{3} \text { TimeTrend }_{t} \text { Exposed }_{k}+\gamma_{1} \text { Race }_{i}+\gamma_{2} \text { Age }_{i} \\
& +\gamma_{3} \text { Insurance }_{i}+\gamma_{4} \text { CharlsonComorbidity }_{i}+\varepsilon_{i c t}
\end{aligned}
$$

Difference-in-differences analysis allows for a natural retrospective experiment while accounting for secular changes by assuming that the control is a valid counterfactual for the exposed group had the policy not been implemented. By showing that prepolicy trends are similar between exposure and control groups, it is reasonable to then consider the control as the counterfactual, as established by published best practices. The parallelism of prepolicy trends can be performed either by graphical inspection or by the following statistical method. Data are restricted to the prepolicy period (before 2012), and a linear TimeTrend variable is used to denote the time interval since the beginning of the study period. Otherwise, the terms are equivalent to those in Equation 1. The difference-in-differences estimator, $\beta_{3}$, is the interaction of the linear time trend and exposure to the policy, measuring any prepolicy divergence between the exposure and control groups. If this term is statistically insignificant, then no prepolicy difference is observed, supporting the control as a valid counterfactual. The prepolicy trends analysis comparing prostate-specific antigen (exposure) versus cholesterol (control) testing in primary care screening is presented in supplemental eAppendix 2. 
2 - Magnani et al

\begin{tabular}{|c|c|c|}
\hline \multicolumn{3}{|c|}{$\begin{array}{l}\text { eAppendix 2. Prepolicy Trends in Prostate-Specific } \\
\text { Antigen and Cholesterol Testing in } \\
\text { the Primary Care Setting }\end{array}$} \\
\hline Age Group & $\begin{array}{c}\text { Trend Difference, } \\
\text { Percentage Points } \\
(95 \% \mathrm{Cl})\end{array}$ & $P$ Value \\
\hline All & $0.6(-0.2$ to 1.4$)$ & .13 \\
\hline $35-54$ y & $0.8(-0.2$ to 1.8$)$ & .13. \\
\hline $55-74$ y & $0.4(-1.0$ to 1.8$)$ & .60 \\
\hline$\geq 75 y$ & $-0.4(-2.2$ to 1.5$)$ & .70 \\
\hline
\end{tabular}

Parallel trends are assessed only in the prepolicy period (2008-2011). Time intervals were defined as calendar years and were evaluated independently for patientlevel eligibility. The trend difference is obtained as the difference-in-differences estimator ( $\beta_{3}$ in Equation 2 in eAppendix 1$)$ for the interaction of the linear time trend and exposure to the policy. When this term is statistically insignificant, there is no observable prepolicy difference. A negative trend difference indicates a decline in the exposure group relative to the control. Adjusted difference-in-differences estimates are corrected for age, Charlson comorbidity score, race, and insurance provider type. Charlson comorbidity score and age were derived on an annual basis. 


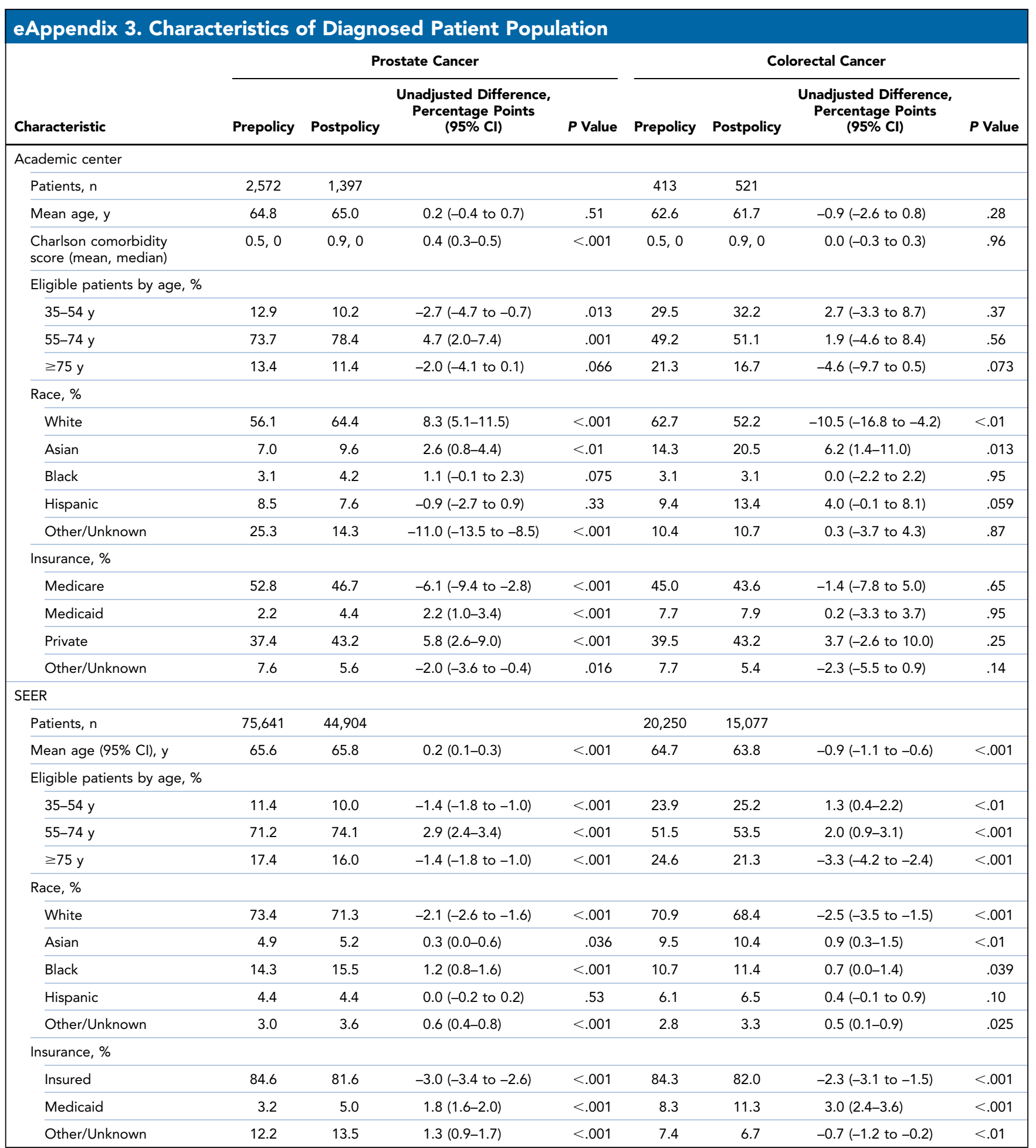

All comparisons are between the prepolicy (2008-2011) and postpolicy (2013-2016) periods. Time intervals were defined as calendar years and evaluated independently for patient-level eligibility. 